\title{
Scalable Distributed Simulation of Large Dense Crowds Using the Real-Time Framework (RTF)
}

\author{
Ole Scharf, Sergei Gorlatch, Felix Blanke, Christoph Hemker, \\ Sebastian Westerheide, Tobias Priebs, Christoph Bartenhagen, \\ Alexander Ploss, Frank Glinka, and Dominik Meilaender \\ University of Münster, Germany \\ gorlatch@uni-muenster.de
}

\begin{abstract}
The simulation of large groups (crowds) of individuals is a complex and challenging task. It requires creating an adequate model that takes into account psychological features of individuals, as well as developing and implementing efficient computation and communication strategies in order to manage the immense computational workload implied by the numerous interactions within a crowd.

This paper develops a novel model for a realistic, real-time simulation of large and dense crowds, focusing on evacuation scenarios and the modeling of panic situations. Our approach ensures that both global navigation and local motion are modeled close to reality, and the user can flexibly change both the simulation environment and parameters at runtime.

Because of the high computation intensity of the model, we implement the simulation in a distributed manner on multiple server machines, using the RTF (Real-Time Framework) middleware. We implement state replication as an alternative to the traditional state distribution via zoning. We show that RTF enables a high-level development of distributed simulations, and supports an efficient runtime execution on multiple servers.
\end{abstract}

\section{Introduction}

The simulation of the behavior of large and dense human crowds is a socially important and technologically challenging task. If attempted with real people, the re-enactment of a scenario such as the evacuation of a fully occupied sports arena poses substantial problems: (1) the costs of involving several thousand people can be enormous; (2) the safety of persons participating in the re-enactment of a potentially life-threatening situation can not be fully guaranteed; (3) simulations involving panics cannot be reproduced at all, since people behave differently when they are aware that the danger is not real; and (4) the inflexibility of reenactments limits the usability: parameters cannot easily be changed, planning is extensive, change of environment is close to impossible. A computer-based simulation overcomes these drawbacks: sophisticated scenarios and large crowds can be set up in reasonable time, simulations can be changed at runtime and repeated with many variations of parameter sets. 
Crowd modeling and simulation is a comparatively young and vigorously studied research area. Purely mathematical approaches or analytic models are not adequate in characterizing the dynamics of a crowd, because it is not simply a collection of individuals, but may exhibit highly complex dynamics due to social and psychological factors. To represent the behavior of a crowd, different kinds of models have been proposed: flow-based, entity-based, and agent-based models.

This paper enhances the agent-based approach as the most accurate one due to the cognitive and reasoning capabilities embedded into the model, in contrast to flow-based models that ignore the individual properties altogether and to entitybased models that express them only in a restricted manner. However, accuracy comes at a price: agent-based models are very time- and space-consuming, thus posing a challenge and an opportunity for parallel and distributed computing. We develop a novel model for computer-based simulations that reproduces the motion of a crowd of individuals by a combination of psychological and geometrical rules with social and physical forces. We describe a new parallelization approach based on state replication rather than on traditional zoning and implement this approach using the Real-Time Framework (RTF) [5] developed at the University of Münster

In Section 2, we extend the recent approach 9112 in which persons in a crowd are regarded as individual entities, so-called agents. We describe how the simulation is distributed among several machines in Section 3 by using a high-level approach based on RTF. We outline our simulation environment and its graphical user interface for interactively changing the simulation at runtime. Section 4 reports first experimental results that demonstrate the scalability of the approach.

\section{The Model for Crowd Simulation}

In developing our model for crowd simulation, we aim at the following properties: (a) capability to simulate the motion of and within large crowds, in particular dense crowds which pose special challenges; (b) high flexibility in the creation of scenarios and complex surrounding areas; (c) possibility to manipulate the simulation interactively at runtime; (d) no inherent design elements in the model that could prevent the distribution or parallelization of computations.

Our approach is based on the HiDAC (High-Density Autonomous Crowds) model 9,12 which is a multi-agent system without a central controlling unit. Each agent corresponds to a simulated person with its own individual behavior.

As illustrated in Figure 1, crowd behavior is computed on two levels:

1. The high-level module simulates behavior from a global perspective. Based on the environment information, the agent navigation within its surroundings is computed and passed on in form of waypoints (intermediate targets), represented by coordinates in virtual space, to the low-level module. 
2. The low-level module determines the agent's perception of its surroundings and derives the necessary motion to reach its current waypoint.

Both modules take into account an agent's physiological and psychological status while at the same time updating it. We made several modifications to the original HiDAC architecture, which are described in the following sections.

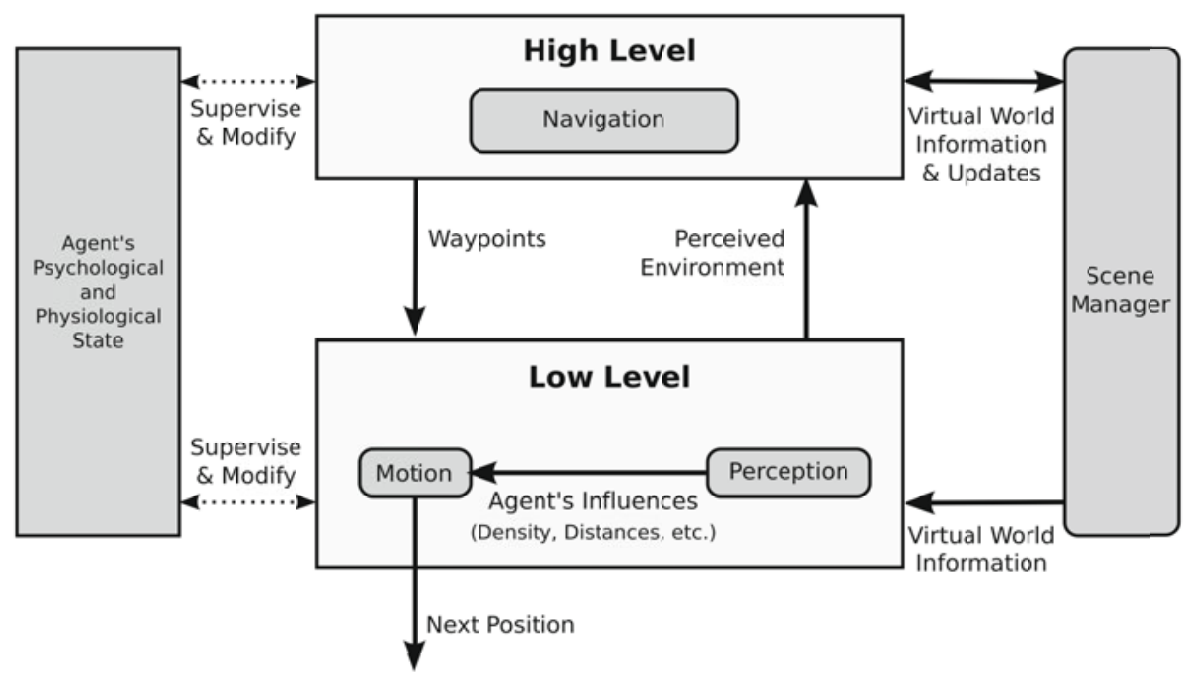

Fig. 1. Modified HiDAC architecture

\subsection{The High-Level Module of the Model}

We use a Cell-Portal-Graph (CPG) to represent the interrelation between rooms and doors of a building used by the crowd as cells and portals correspondingly. Rooms carry the information about enclosing walls and agents inside. Nodes represent rooms, edges represent their connections, the portals. In the indoor scenarios, the latter are commonly doors or bottlenecks, therefore inducing the generalized term 'door' for that kind of connection. Our CPG implementation allows for multiple edges between any two nodes. Figure 2 shows an example translation of an indoor scenario with eight rooms and ten doors into a CPG.

Agents pursue a global goal, e.g., leaving the building, by following a sequence of waypoints at those doors that lead to the exit. Agents react dynamically to changes in their environment (e.g., if a door is interactively closed during simulation) and can select alternative routes. As an extension to HiDAC, we implemented the capability to subdivide overlarge simulated rooms on graph level. This allows us to balance the CPG, thus increasing the performance and facilitating the modeling of route-selection preferences. By permitting arbitrary orientation and quantities of walls, we enable a flexible, unrestricted modeling 


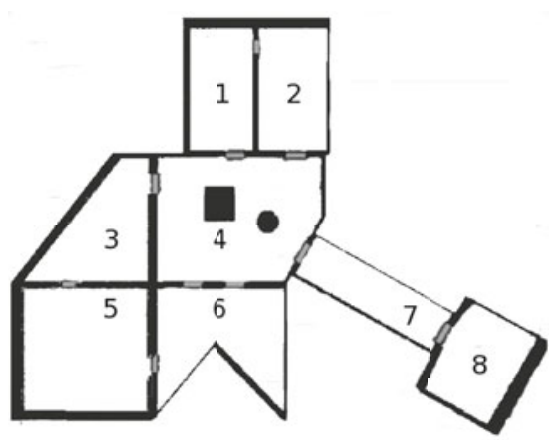

(a) A building with rooms and doors.

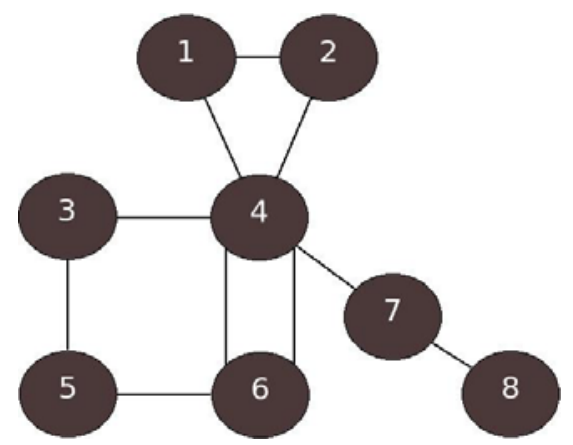

(b) The model of the building as CPG.

Fig. 2. Translation of a complex building scenario into a $\mathrm{CPG}$

of the surroundings and an improved waypoint system, empowering agents to navigate even in very complex environments.

We use the Boost Graph Library (BGL) 3] for implementing the CPG, building upon the library's class adjacency_list which offers methods for accessing specified nodes or edges. The room-related data of nodes is held in a std::vector list, the door-related data of the edges is held in a std::multiset, thus allowing direct access to looked-up edges between known nodes by implicit sorting.

Pathfinding. If the surroundings are known, the agent's pathfinding is reduced to finding the shortest path from its starting room to its target room, i.e. between the corresponding nodes in the graph. Finding this path is strongly influenced by the weighted edges in our graph implementation: Crowding agents in front of a door add to the weight of the associated edge in the graph. As each agent increases the weight by the amount of space it requires (in our model, this is represented by a circular area defined by the agent's individual radius), the weight of an edge can thus be understood as a measure of the occupied space in front of the door. With $d_{a}$ denoting the shortest distance of an agent to the door, $w$ the current weight, and $r_{a}$ the agent's radius, the agent increases the weight by $2 \cdot r_{a}$, if $d_{a}<w+r_{a}$.

An agent is excluded from the weight calculation if it has passed through the door or decides to follow an alternative route. Utilizing the Dijkstra's algorithm (dijkstra_shortest_paths in BGL), a list of $N$ nodes and corresponding rooms is generated which the agent has to traverse on its way. $\operatorname{In} \mathcal{O}(|N| \cdot \log |N|)$ time, this algorithm yields a minimal path w.r.t. the weighted sum of its edges.

Alternative routes. Two kinds of events can trigger the necessity to reconsider the currently followed path of an agent: A door it was going to pass through turns out to be closed or jammed by other agents. Encountered closed doors in immediate perception range, as seen in Figure 3, are kept in the agent's memory and trigger a re-run of pathfinding. When an agent wishes to pass through an open, though jammed door, this agent's impatience is the decisive factor. 

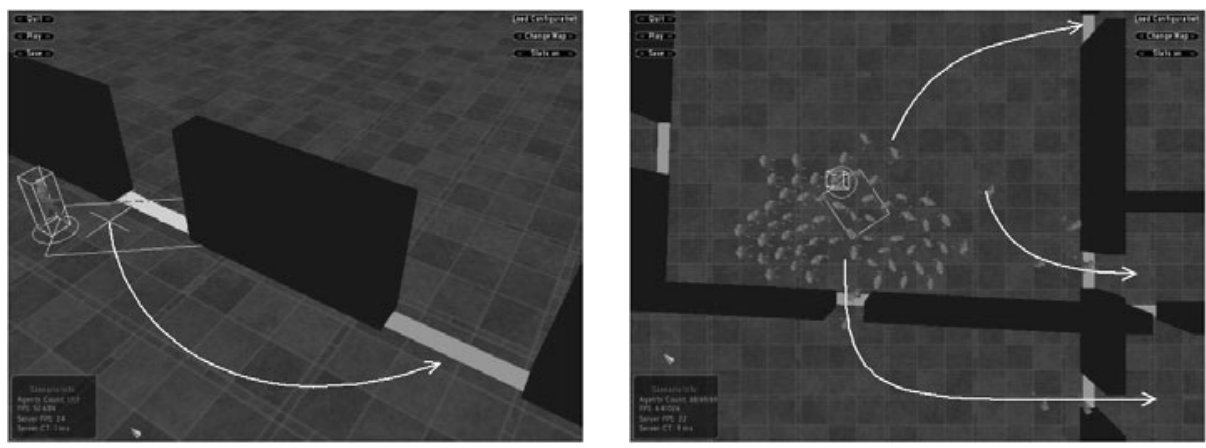

Fig. 3. Agents take alternative routes because of a closed (left) or jammed door (right)

Collision Detection and Assisting/Local Waypoints. Our model improves upon HiDAC by circumnavigating the hindering parts of a room's geometry obstructing an agent's way. To do so, we introduce assisting waypoints, dynamically set within a room itself, in contrast to the waypoints used in the global navigation. An assisting local waypoint is derived in order to guide the agent around the obstacle, taking into account all walls an agent is currently perceiving.

The derivation is illustrated in Figure 4. Let $n_{w}$ be the normal vector, $o_{w}$ the orientation vector, $S$ and $E$ the starting point and the endpoint of a wall, correspondingly. Moreover, let $T$ be the agent's current (global or local) target, $P$ its position, $d_{w}$ the shortest distance to the wall and $L$ a potential local waypoint at one end of the wall. Then a sequence of tests is performed (e.g., on which side of the wall is the agent positioned, is there an intersection between the direct path and the wall, is this the closest wall, etc.) which allow the agent to keep its motion towards the overall target, e.g., an exit of the building.

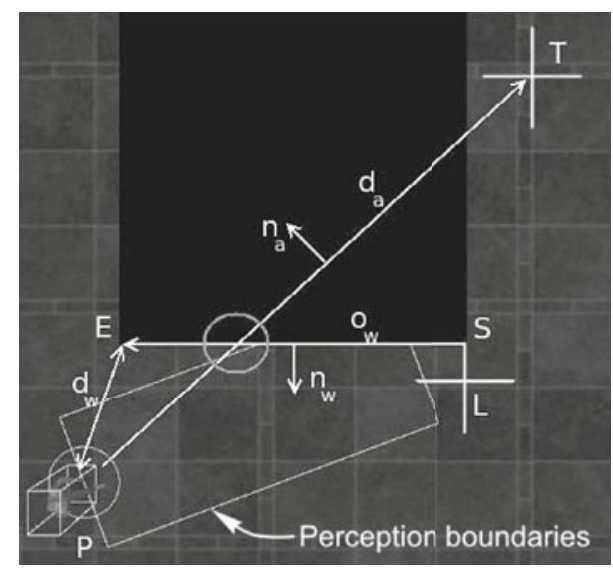

Fig. 4. Boundaries of agent's perception and assisting waypoint L 


\subsection{The Low-Level Module: Perception and Motion}

While the high-level module is responsible for global navigation and determining waypoints, the low-level module deals with the simulation of perception and local motion of an agent, as illustrated in Figure 1.

Our implementation extends and modifies the original HiDAC 9 as follows. Local motion is based on a combination of psychological, physiological and geometrical rules with physical forces. Agent movement derives from the weighted sum of these forces, in particular representing the agent's motion-related goals: reaching its current waypoint, avoiding collision with other agents, obstacles and walls, collision handling, and assuming a certain motion inertia towards maintaining its current direction of movement. Agents possess an individual perception range which is of significance for the computational distribution over a network: collision avoidance takes place for agents, obstacles and walls within this perception range only. Collision handling is enforced regardless of the perception range. While collision avoidance is only applied to the room the agent is currently residing in, collision handling additionally accounts for any agents from other rooms passing through connecting doors. Thereby, we prevent unnatural oscillating behavior of agents in crowded spaces. Furthermore, the most important behavioral patterns are simulated: queuing, pushing through crowds, falling down, panicking with panic propagation, and anticipatory agents considering crowd density by means of dynamically adjusting their perception range.

On top of our own implementation of structural elements described in [9], we introduced a more situation-aware, intelligent collision avoidance, as well as modifications regarding the avoidance of walls. Another novel feature is that agents look ahead through doors into other rooms when accounting for elements to avoid. The handling of collisions has also been modified to improve agent behavior in collision loops (when agents repeat the same steps again and again, that might eventually lead to a collision).

\section{Distributed Multi-server Implementation}

High-performance simulation is a prerequisite for a broad class of immersive applications where the user participates in the simulation from the first person perspective. We achieve this by employing a multi-server distribution approach and by providing a comfortable interface for interactions. For distributing the computations over a network of computers, we use the Real-Time Framework (RTF) [5]. The RTF middleware was originally designed to support a high-level development of Internet-based interactive applications like multi-player online computer games. In our simulation system, RTF is responsible for efficient parallel computations, communication and synchronization.

\subsection{Serialization and Area of Interest Management}

The distribution of tasks between the servers and clients in our system is as follows: a client is processing user interactions, it relates them to the simulation, 
and renders a $3 \mathrm{D}$ visualization, while the computation of the actual simulation state takes place in a distributed manner over multiple servers.

This task separation and distributed computations require a communication interface for transferring data between clients and servers throughout the system. The Real-Time Framework offers an efficient and comfortable means to implement the data transfer: in particular, it supports automatic serialization of objects, i.e. transforming them into a a network-transmittable form. User-defined serialization is offered optionally.

Area of Interest (AoI) Management describes the process of distinguishing between relevant and irrelevant information within the simulation process. We apply the AoI concept of RTF [5] on both client- and server-side, dismissing some unnecessary data transfers in order to reduce network traffic. The cost of a potential transfer is weighed against its benefit: (a) information is rated valuable for a client if it makes a noticeable difference to the visualization; (b) information is rated valuable for a server if it supports a consistent simulation state throughout the distributed system. Such AoI management allows us to limit visualization updates transmitted over the network to only those agents which reside inside the user's field of view, thus saving resources on the servers and the network bandwidth.

\subsection{Distribution: Zoning and Replication}

The intuitive technique traditionally used in many distributed applications is 'zoning': the environment is split into disjoint zones, in which computations are handled by different servers, see Fig. 5 (left). When a moving entity (agent or avatar) leaves a zone and enters another zone, this changes the server's responsibility for this entity.

For crowd simulations, the 'zoning' approach has several drawbacks. First, agent interaction over zone borders is prevented, since information is exclusively available only to one responsible server. Thus, an agent cannot make a decision based on observing other remote agents, which is often necessary in practical scenarios. Moreover, when simulating dense crowds, we cannot distribute the
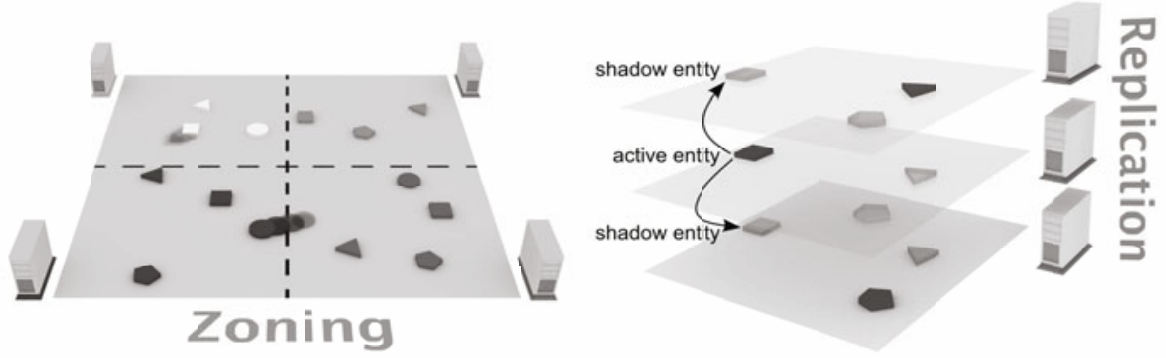

Fig. 5. Concepts of 'zoning' (left) and 'replication' (right) 
computational workload where it is especially needed: zone borders can only be placed in sparsely populated areas, thus eventually leaving the simulation of a very densely populated area to one server. Finally, strict separation of data among servers requires the client, responsible for visualization, to communicate frequently with every single server in order to render a complete picture of the simulation state.

The novelty of this paper is to explore the use of 'replication' rather than 'zoning' for computation distribution. Replication means that each server holds the complete simulation data, see Fig. 5 (right). Each server is computing updates only for its so-called active agents; all other agents are called shadowed on this server, and their updates are computed by other servers (every agent is active on exactly one server) and received from them. This allows us to distribute the workload evenly between servers, even in densely crowded scenarios, without hindering agent interaction as with 'zoning'. Additionally, a client now only needs to connect to one server to receive a complete picture of the simulation state for visualization. Replication in our implementation is implemented using RTF which supports both replication and zoning and advanced combinations thereof (the latter will be studied in our future work). The simulation environment is described on a high level of abstraction in an RTF-specific 'map' which determines the distribution of geometrical space on available servers. Our current system employs a single area replicated over the network: each server comes with its own HiDAC unit. Using mechanisms offered by RTF, agents can be added to a unit, removed from it, and migrated to a different unit at runtime.

Agents, doors and obstacles are initialized on one server and subsequently replicated on the others. For example, a newly created obstacle on server A, at first only exists inside the HiDAC unit on server A and inside its RTF Communication and Computation Parallelization (CCP) Module. RTF automatically recreates this obstacle in the $\mathrm{CCP}$ Module of server $\mathrm{B}$, which is then to be inserted into server B's HiDAC unit in the final step of the replication process. RTF manages replication across all participating servers, such that eventually each server keeps an instance of the modeled obstacle. In the terminology of RTF, the obstacle is 'active' on server A, whereas it is 'shadowed' on all other servers. This means that server A has sole authority for manipulating this obstacle's state, while the remaining servers receive updates from A on state changes.

An essential feature of replication is that the state of all agents must be known to all servers. Thus, servers need to update the state of their active entities on all other servers. These state updates are automatically performed by RTF and comprise three main steps: 1) serialize agent states and prepare messages, 2) transmit messages over the network, and 3) de-serialize agent states. For a particular server, the complexity of these steps depends linearly on two factors: the total number of agents and the number of servers. To reduce the overhead of replication, RTF employs several optimizations. The serialization of active agent states into a network-transmittable form is performed exactly once per tick for each modified agent. To minimize the number of network packets, RTF aggregates multiple agent updates to a single packet depending on the maximum 
transfer unit of the underlying network. RTF decouples de-/ serialization of state updates and actual transmission over the network: while the de-/ serialization of agent states is synchronized with the simulation process in order to prevent concurrency issues, the actual network transmission is handled by RTF asynchronously. Hence, the transmission usually does not add up to the processing time of the application. The amount of transmitted messages could be further reduced using multicast communication which, however, is not always available in distributed systems operated over the Internet.

\subsection{Visualization}

We implement the crowd simulation as a real-time system with an intuitive $3 \mathrm{D}$ representation of the current simulation state which facilitates the evaluation of the simulation state and results by the user. The users can interactively change a running or a paused simulation and manipulate simulation parameters. The parameters currently span 18 different agent-specific attributes, ranging from simple radius and maximum velocity to more advanced right preference angle and attractor weight modifiers. The fine-grain control over every single simulated entity is one of the main advantages of agent-based simulations, and it is made readily available in the user interface of our system.

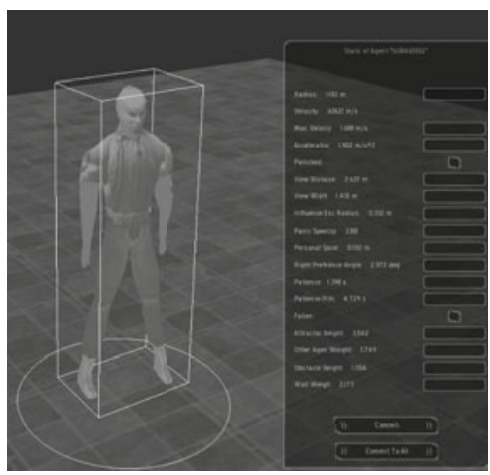

Fig. 6. Example of changing the simulation parameters at runtime

In our implementation, we used the following free software: OGRE (ObjectOriented Graphics Rendering Engine, [10]) for three-dimensional visualization, CEGUI (Crazy Eddie's GUI, [4]) as graphical user interface, and OIS (Object Oriented Input System, [1]) for handling mouse and keyboard. Also, we employ Art of Illusion 3D-modeling software 11 for scenario-ready representations of environmental surroundings, with arbitrary orientation/quantities of walls.

\section{Experimental Results}

In order to assess the performance of our simulation system, we conducted a series of tests in a high-load setup which emphasized those elements of a simulation 
scenario that lead to bottlenecks in the system's performance. We studied a complex indoor environment with many rooms and one large, unobstructed area, which is much more challenging than the simpler scenarios studied previously. While agents hidden from another agent's sight can be disregarded in many calculations, open space takes away this potential performance gain. Also, our testing scenario's setup ensures permanent agent movement because this induces additional computational workload. E.g., a scenario with 400 stationary agents might require less computing power than a scenario with 200 moving agents. Measurements were conducted on a local network of common desktop PCs (servers) at the University of Muenster, with identical hardware characteristics: CPU: Intel(R) Core(TM) 2 Duo CPU E6750 @ 2.66 GHz; Memory: 4 GB; Network connection: 100 Mbit / half duplex. The maximum number of such simultaneously used servers in our experiments was 12 .

The measured value in the experiments is the rate of simulation frames per second (fps) successfully calculated on a server. By always choosing the value of the weakest system-wide server as the indicator for overall performance, we again followed the high-load approach. Measurements were done in the following manner: First, the server environment was prepared, comprising 1, 2, 4, 6, 8, 10, or 12 servers, with a fair assignment of agents to servers. Then, the test scenario was populated with 20 agents. After 1 minute runtime, the server simulation speed was measured. Subsequently, all agents were removed, the simulation then again was populated with 40 to 400 agents, with step 20 agents, and measurements were taken again after 1 minute.

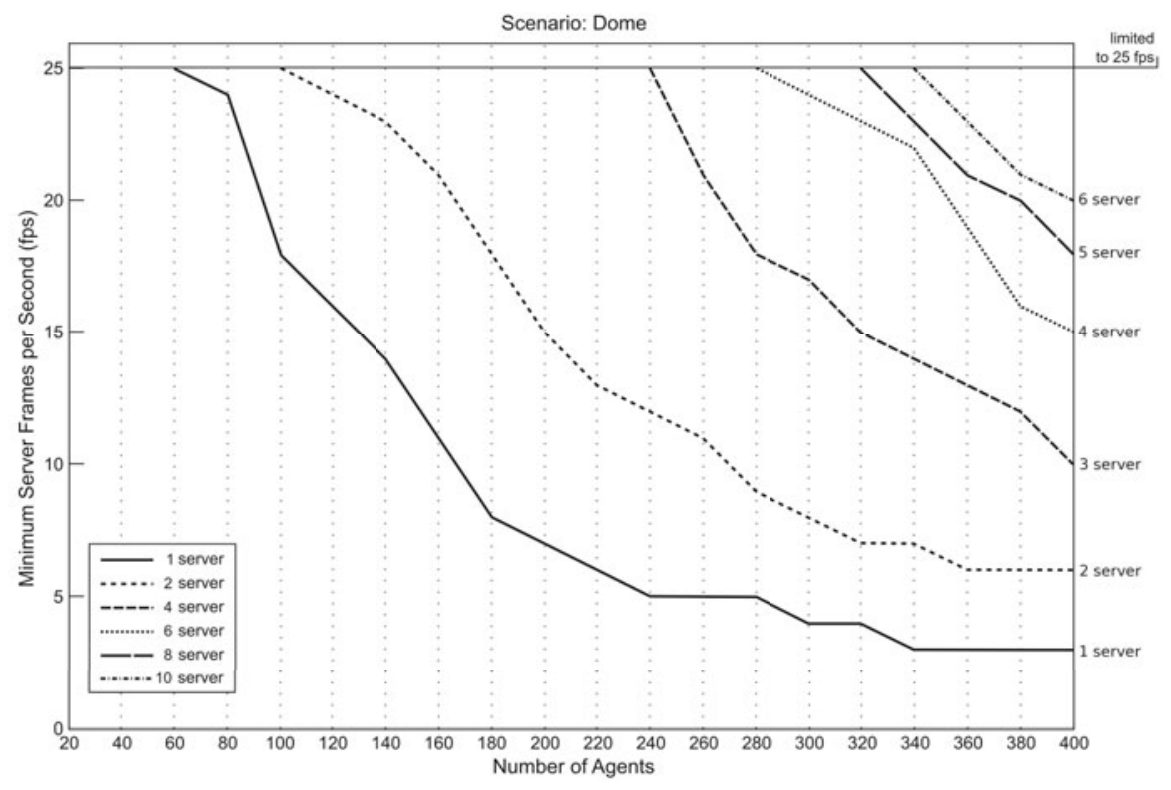

Fig. 7. Measurements of the simulation speed on 1 to 10 servers 
Our series of tests with the specifically designed evacuation scenario for the St. Paulus Cathedral in Muenster (a medieval building of about $5000 \mathrm{sqm}$ with a complex system of doors) produce the results shown in Figure7. We observe that an increase in the number of servers allows for the simulation of more agents, or, at a fixed number of agents, increases the rate of simulation in fps. A value of $10 \mathrm{fps}$ is an empirically found threshold to ensure correct calculations in our implementation: rate $\ll 10$ fps may lead to calculation errors, e.g., agents passing through walls. As shown in Figure 7 four servers already suffice to achieve this threshold for up to 395 simulated agents. Regarding scalability, one server can simulate 170 agents at $10 \mathrm{fps}$, whereas two servers manage 280 agents at the same frame rate (an increase of $64 \%$ ), and four servers can increase this number further to 395 (132\%).

\section{Conclusion and Future Work}

The main contributions of this paper are two-fold:

- We developed a novel agent-based model for dense crowds by extending and modifying the HiDAC approach 12 based on the previous work 6] and improving the models suggested in 27]. As compared with the original HiDaC model, our approach is better suitable for complex indoor scenarios with many rooms and also large, unobstructed areas, which together lead to highly intensive calculations regarding collision detection and AoI management. We designed and implemented a distributed simulation system based on this model. According to the recent comprehensive surveys of the crowd simulation area 813, our system can be classified as a medium-size one, with a short- to medium-term time scale of underlying processes. The distributed nature of our system, together with its high scalability, allows us to challenge the "huge-sized" barrier which is currently held almost exclusively by flow-based approaches. In comparison with them, our system provides definite advantages regarding such critical features as flexibility (interactive changes at runtime), extensibility (accommodating new behavioral factors) and efficiency (real-time response) and, most importantly, regarding the scalability over the number of servers used for simulation.

- We demonstrated that our Real-Time Framework (RTF) [5], originally created for interactive online applications like multi-player online games, is very suitable for the area of distributed simulation: (a) RTF facilitates a highlevel approach to system design, by automatizing many important functions: serialization, distribution, and resource migration; (b) RTF supports highperformance simulation at runtime and ensures high scalability.

Our work improves on previous approaches which are mostly limited to single workstations, post-simulation evaluation only, or to models abstracting from the individual towards more coarse-grained entities due to the performance issues. Our system offers a graphical user interface for evaluation and interactive change 
of simulation parameters at runtime, thus setting a cornerstone for future applications targeting first-person perspective immersion into large agent-based simulations.

Our future work will improve on both our model and simulation system: introducing fault-tolerance features for the case of a machine failure, combining the replication approach described here with the traditional zoning, etc. With our approach, factored-in psychological and physiological attributes can be easily extended, e.g., embedding the imitation of behavior of others while being part of a crowd (commonly observed in real life situations), as well as physical handicaps or exhaustion phenomena.

\section{References}

1. Art of Illusion, Open source 3D modeling and rendering studio, http://www.artofillusion.org/

2. Batty, M.: Polynucleated Urban Landscapes. Urban Stud. 38(4), 635-655 (2001), http://usj.sagepub.com/cgi/content/abstract/38/4/635

3. Boost Graph Library, version 1.34, http://www.boost.org/

4. Crazy Eddie's GUI, http://www.cegui.org.uk/

5. Glinka, F., Ploss, A., Gorlatch, S., Müller-Iden, J.: High-level development of multiserver online games. International Journal of Computer Games Technology (5), 1-16 (2008)

6. Helbing, D., Buzna, L., Johansson, A., Werner, T.: Self-organized pedestrian crowd dynamics: Experiments, simulations, and design solutions. Transportation Science 39(1), 1-24 (2005)

7. Hughes, R.L.: The Flow of Human Crowds. Annual Review of Fluid Mechanics 35, 169-182 (2003)

8. Lozano, M., Morillo, P., Ordua, J., Cavero, V., Vigueras, G.: A new system architecture for crowd simulation. Journal of Network and Computer Applications 32(2), 474-482 (2009)

9. Pelechano, N., Allbeck, J., Badler, N.: Controlling individual agents in high-density crowd simulation. In: ACM SIGGRAPH / Eurographics Symposium on Computer Animation, San Diego, USA (August 2007)

10. Object-Oriented Graphics Rendering Engine, http://www.ogre3d.org/

11. Object-Oriented Input System, http://sourceforge.net/projects/wgois/

12. Pelechano, N., Allbeck, J.M., Badler, N.I.: Virtual crowds: Methods, simulation, and control. Synthesis Lectures on Computer Graphics and Animation 3(1), 1-176 (2008)

13. Zhou, S., Chen, D., Cai, W., Lyo, L., Yoke, M., Hean, L., Tian, F., Wee Sze Ong, D., Su-Han Tay, V., Hamilton, B.D.: Crowd modeling and simulation technologies. ACM Transactions on Modeling and Computer Simulation (2009) 